\title{
SOME RESULTS ON UNIQUENESS OF ENTIRE FUNCTIONS CONCERNING DIFFERENCE POLYNOMIALS
}

\author{
PULAK SAHOO AND GURUDAS BISWAS
}

\begin{abstract}
In the paper we use the notion of weakly weighted sharing and relaxed weighted sharing to investigate the uniqueness problems when two difference products of entire functions share a small function. The results of the paper improve and extend some recent results due to the present first author [Commu. Math. Stat., 3 (2015), 227-238].
\end{abstract}

\section{Introduction, Definitions and Results}

In this paper, by meromorphic function we will always mean meromorphic function in the complex plane. We assume that the reader is familiar with the basic notions of Nevanlinna value distribution theory (see [9], [12] and [24]). For a nonconstant meromorphic function $f$, we denote by $T(r, f)$ the Nevanlinna characteristic function of $f$ and by $S(r, f)$ any quantity satisfying $S(r, f)=o\{T(r, f)\}$ as $r \longrightarrow \infty$ outside of an exceptional set of finite linear measure. We say that $\alpha(z)$ is a small function of $f$, if $\alpha(z)$ is a meromorphic function satisfying $T(r, \alpha(z))=S(r, f)$.

Let $k$ be a positive integer or infinity and $a \in \mathbb{C} \cup\{\infty\}$. Set $E(a, f)=\{z: f(z)-a=0\}$, where a zero with multiplicity $k$ is counted $k$ times. If the zeros are counted only once, then we denote the set by $\bar{E}(a, f)$. Let $f$ and $g$ be two nonconstant meromorphic functions. If $E(a, f)=E(a, g)$, then we say that $f$ and $g$ share the value $a \mathrm{CM}$ (counting multiplicities). If $\bar{E}(a, f)=\bar{E}(a, g)$, then we say that $f$ and $g$ share the value $a$ IM (ignoring multiplicities). We denote by $E_{k)}(a, f)$ the set of all $a$-points of $f$ with multiplicities not exceeding $k$, where an $a$-point is counted according to its multiplicity. Also we denote by $\bar{E}_{k)}(a, f)$ the set of distinct $a$-points of $f$ with multiplicities not exceeding $k$. Throughout the paper, we denote by $\rho(f)$ the order of $f$ (see [9], [12] and [24]). In addition, we need the following definitions.

Definition 1 ([10]). Let $a \in \mathbb{C} \cup\{\infty\}$. We denote by $N(r, a ; f \mid=1)$ the counting function of simple $a$-points of $f$. For a positive integer $k$ we denote by $N(r, a ; f \mid \leq k)$ the counting function of

Received April 25, 2016, accepted January 19, 2018.

2010 Mathematics Subject Classification. Primary 30D35.

Key words and phrases. Uniqueness, entire function, small function, difference polynomial.

Corresponding author: Pulak Sahoo. 
those $a$-points of $f$ (counted with proper multiplicities) whose multiplicities are not greater than $k$. By $\bar{N}(r, a ; f \mid \leq k)$ we denote the corresponding reduced counting function. Analogously we can define $N(r, a ; f \mid \geq k)$ and $\bar{N}(r, a ; f \mid \geq k)$.

Definition 2 ([11]). Let $k$ be a positive integer or infinity. We denote by $N_{k}(r, a ; f)$ the counting function of $a$-points of $f$, where an $a$-point of multiplicity $m$ is counted $m$ times if $m \leq k$ and $k$ times if $m>k$. Then

$$
N_{k}(r, a ; f)=\bar{N}(r, a ; f)+\bar{N}(r, a ; f \mid \geq 2)+\cdots+\bar{N}(r, a ; f \mid \geq k) .
$$

Clearly $N_{1}(r, a ; f)=\bar{N}(r, a ; f)$.

Definition 3. Let $a \in \mathbb{C} \cup\{\infty\}$. We denote by $N_{E}(r, a ; f, g)\left(\bar{N}_{E}(r, a ; f, g)\right)$ by the counting function (reduced counting function) of all common zeros of $f-a$ and $g-a$ with the same multiplicities and by $N_{0}(r, a ; f, g)\left(\bar{N}_{0}(r, a ; f, g)\right)$ the counting function (reduced counting function) of all common zeros of $f-a$ and $g-a$ ignoring multiplicities. If

$$
\bar{N}(r, a ; f)+\bar{N}(r, a ; g)-2 \bar{N}_{E}(r, a ; f, g)=S(r, f)+S(r, g),
$$

then we say that $f$ and $g$ share the value $a$ "CM". If

$$
\bar{N}(r, a ; f)+\bar{N}(r, a ; g)-2 \bar{N}_{0}(r, a ; f, g)=S(r, f)+S(r, g),
$$

then we say that $f$ and $g$ share the value $a$ "IM".

Definition 4 ([14]). Let $f$ and $g$ share the value $a$ "IM" and $k$ be a positive integer or infinity. Then $\bar{N}_{k)}^{E}(r, a ; f, g)$ denotes the reduced counting function of those $a$-points of $f$ whose multiplicities are equal to the corresponding $a$-points of $g$, and both of their multiplicities are not greater than $k \cdot \bar{N}_{(k}^{0}(r, a ; f, g)$ denotes the reduced counting function of those $a$-points of $f$ which are $a$-points of $g$, and both of their multiplicities are not less than $k$.

We now introduce the following definition of weakly weighted sharing which is a scaling between sharing IM and sharing CM.

Definition 5 ([14]). Let $a \in \mathbb{C} \cup\{\infty\}$ and $k$ be a positive integer or infinity. If

$$
\begin{aligned}
& \bar{N}(r, a ; f \mid \leq k)-\bar{N}_{k)}^{E}(r, a ; f, g)=S(r, f), \\
& \bar{N}(r, a ; g \mid \leq k)-\bar{N}_{k)}^{E}(r, a ; f, g)=S(r, g), \\
& \bar{N}(r, a ; f \mid \geq k+1)-\bar{N}_{(k+1}^{0}(r, a ; f, g)=S(r, f), \\
& \bar{N}(r, a ; g \mid \geq k+1)-\bar{N}_{(k+1}^{0}(r, a ; f, g)=S(r, g),
\end{aligned}
$$

or if $k=0$ and 


$$
\begin{aligned}
& \bar{N}(r, a ; f)-\bar{N}_{0}(r, a ; f, g)=S(r, f), \\
& \bar{N}(r, a ; g)-\bar{N}_{0}(r, a ; f, g)=S(r, g),
\end{aligned}
$$

then we say that $f$ and $g$ share the value $a$ weakly with weight $k$ and we write $f$ and $g$ share " $(a, k)$ ".

In 2007, A. Banerjee and S. Mukherjee [1] introduced a new type of sharing known as relaxed weighted sharing, weaker than weakly weighted sharing and is defined as follows.

Definition 6 ([1]). We denote by $\bar{N}(r, a ; f|=p ; g|=q)$ the reduced counting function of common $a$-points of $f$ and $g$ with multiplicities $p$ and $q$ respectively.

Definition 7 ([1]). Let $a \in \mathbb{C} \cup\{\infty\}$ and $k$ be a positive integer or infinity. Suppose that $f$ and $g$ share the value $a$ "IM". If for $p \neq q$,

$$
\sum_{p, q \leq k} \bar{N}(r, a ; f|=p ; g|=q)=S(r),
$$

then we say that $f$ and $g$ share the value $a$ with weight $k$ in a relaxed manner and in that case we write $f$ and $g$ share $(a, k)^{*}$.

Many research works on entire and meromorphic functions whose differential polynomials share certain value, small function or fixed points have been done by many mathematicians in the world (see [5], [6], [15], [20], [22], [23]). Recently, value distribution in difference analogue has become a subject of great interest among the researchers. In 2006, R.G. Halburd and R.J. Korhonen [7] established a version of Nevanlinna theory based on difference operators. The difference logarithmic derivative lemma, given by R.G. Halburd and R.J. Korhonen [8] in 2006, Y.M. Chiang and S.J. Feng [4] in 2008 plays an important role in considering the difference analogues of Nevanlinna theory. With the development of difference analogue of Nevanlinna theory, many mathematicians paid their attention on the distribution of zeros of different types of difference polynomials. In 2007, I. Laine and C.C. Yang [13] proved the following result.

Theorem A. Let $f(z)$ be a transcendental entire function of finite order and $\eta$ be a nonzero complex constant. Then for $n \geq 2, f^{n}(z) f(z+\eta)$ assumes every nonzero value $a \in \mathbb{C}$ infinitely often.

We recall the following two examples.

Example 1 ([13]). Let $f(z)=1+e^{z}$. Then $f(z) f(z+\pi i)-1=-e^{2 z}$ has no zeros. This shows that Theorem A does not hold if $n=1$. 
Example 2 ([17]). Let $f(z)=e^{-e^{z}}$. Then $f^{2}(z) f(z+\eta)-2=-1$ and $\rho(f)=\infty$, where $\eta$ is the nonzero constant satisfying $e^{\eta}=-2$. Evidently, $f^{2}(z) f(z+\eta)-2$ has no zeros. This shows that Theorem A does not remain valid if $f$ is of infinite order.

In 2010, X.G. Qi, L.Z. Yang and K. Liu [19] proved the following uniqueness result corresponding to Theorem A.

Theorem B. Let $f(z)$ and $g(z)$ be two transcendental entire functions offinite order, and $\eta$ be a nonzero complex constant, and let $n \geq 6$ be an integer. If $f^{n}(z) f(z+\eta)$ and $g^{n}(z) g(z+\eta)$ share $1 C M$, then either $f g=t_{1}$ or $f=t_{2} g$ for some constants $t_{1}$ and $t_{2}$ satisfying $t_{1}^{n+1}=t_{2}^{n+1}=1$.

In the same year J.L. Zhang [25] considered the zeros of one certain type of difference polynomial and proved the following result.

Theorem C. Let $f(z)$ be a transcendental entire function of finite order, $\alpha(z)(\not \equiv 0)$ be a small function with respect to $f(z)$ and $\eta$ be a nonzero complex constant. If $n \geq 2$ is an integer then $f^{n}(z)(f(z)-1) f(z+\eta)-\alpha(z)$ has infinitely many zeros.

In the same paper the author also proved the following uniqueness result.

Theorem D. Let $f(z)$ and $g(z)$ be two transcendental entire functions offinite order, and $\alpha(z)(\not \equiv$ 0 ) be a small function with respect to both $f(z)$ and $g(z)$. Suppose that $\eta$ is a nonzero complex constant and $n \geq 7$ is an integer. If $f^{n}(z)(f(z)-1) f(z+\eta)$ and $g^{n}(z)(g(z)-1) g(z+\eta)$ share $\alpha(z)$ $C M$, then $f(z)=g(z)$.

Naturally one may ask the following question.

Question 1. Is it possible to relax the nature of sharing the small function in Theorem D?

In 2014, using the idea of weakly weighted sharing and relaxed weighted sharing, C. Meng [18] proved the following results which improve and supplement Theorem D in different directions.

Theorem E. Let $f(z)$ and $g(z)$ be two transcendental entire functions of finite order, and $\alpha(z)(\not \equiv$ $0, \infty)$ be a small function with respect to both $f(z)$ and $g(z)$. Suppose that $\eta$ is a nonzero complex constant, and $n \geq 7$ is an integer. If $f^{n}(z)(f(z)-1) f(z+\eta)$ and $g^{n}(z)(g(z)-1) g(z+\eta)$ share “ $(\alpha, 2) "$, then $f(z) \equiv g(z)$.

Theorem F. Let $f(z)$ and $g(z)$ be two transcendental entire functions of finite order, and $\alpha(z)(\not \equiv$ $0, \infty)$ be a small function with respect to both $f(z)$ and $g(z)$. Suppose that $\eta$ is a nonzero complex constant and $n \geq 10$ is an integer. If $f^{n}(z)(f(z)-1) f(z+\eta)$ and $g^{n}(z)(g(z)-1) g(z+\eta)$ share $(\alpha, 2)^{*}$, then $f(z) \equiv g(z)$. 
Theorem G. Let $f(z)$ and $g(z)$ be two transcendental entire functions offinite order, and $\alpha(z)(\not \equiv$ $0, \infty)$ be a small function with respect to both $f(z)$ and $g(z)$. Suppose that $\eta$ is a nonzero complex constant and $n \geq 16$ is an integer. If $\bar{E}_{2)}\left(\alpha(z), f^{n}(z)(f(z)-1) f(z+\eta)\right)=\bar{E}_{2)}\left(\alpha(z), g^{n}(z)(g(z)-\right.$ 1) $g(z+\eta)$ ), then $f(z) \equiv g(z)$.

Observing the above results the following question is inevitable.

Question 2. What can be said about the relationship between two entire functions $f$ and $g$ if one replace $f^{n}(z)(f(z)-1) f(z+\eta)$ by $f^{n}(z)\left(f^{m}(z)-1\right) f(z+\eta)$ in Theorems E - G where $m(\geq 1)$ is any integer?

In recent, the present first author [21] answered the above question and proved the following results which generalize Theorems E-G.

Theorem H. Let $f(z)$ and $g(z)$ be two transcendental entire functions offinite order, and $\alpha(z)(\not \equiv$ $0, \infty)$ be a small function of both $f(z)$ and $g(z)$. Suppose that $\eta$ is a nonzero complex constant, $n$ and $m(\geq 1)$ are integers such that $n \geq m+6$. If $f^{n}(z)\left(f^{m}(z)-1\right) f(z+\eta)$ and $g^{n}(z)\left(g^{m}(z)-\right.$ 1) $g(z+\eta)$ share “ $(\alpha(z), 2)$ ", then $f(z) \equiv \operatorname{tg}(z)$ where $t^{m}=1$.

Theorem I. Let $f(z)$ and $g(z)$ be two transcendental entire functions of finite order, and $\alpha(z)(\not \equiv$ $0, \infty)$ be a small function of both $f(z)$ and $g(z)$. Suppose that $\eta$ is a nonzero complex constant, $n$ and $m(\geq 1)$ are integers such that $n \geq 2 m+8$. If $f^{n}(z)\left(f^{m}(z)-1\right) f(z+\eta)$ and $g^{n}(z)\left(g^{m}(z)-\right.$ 1) $g(z+\eta)$ share $(\alpha(z), 2)^{*}$, then $f(z) \equiv \operatorname{tg}(z)$ where $t^{m}=1$.

Theorem J. Let $f(z)$ and $g(z)$ be two transcendental entire functions of finite order, and $\alpha(z)(\not \equiv$ $0, \infty)$ be a small function of both $f(z)$ and $g(z)$. Suppose that $\eta$ is a nonzero complex constant, $n$ and $m(\geq 1)$ are integers such that $n \geq 4 m+12$. If $\bar{E}_{2)}\left(\alpha(z), f^{n}(z)\left(f^{m}(z)-1\right) f(z+\eta)\right)=$ $\bar{E}_{2)}\left(\alpha(z), g^{n}(z)\left(g^{m}(z)-1\right) g(z+\eta)\right)$, then $f(z) \equiv \operatorname{tg}(z)$ where $t^{m}=1$.

Regarding Theorems H-J, one may ask the following question which is the motivation of the present paper.

Question 3. What can be said about the entire functions $f$ and $g$ if we consider the difference polynomials of the form $\left(f^{n}(z)\left(f^{m}(z)-1\right) f(z+\eta)\right)^{(k)}$ where $k(\geq 0)$ is an integer?

In the paper, our main purpose is to find out the possible answer of the above question. We prove following three theorems which improve and extend Theorems H-J respectively. The following theorems are the main results of the paper.

Theorem 1. Let $f(z)$ and $g(z)$ be two transcendental entire functions of finite order, and $\alpha(z)(\not \equiv$ $0, \infty)$ be a small function of both $f(z)$ and $g(z)$ with finitely many zeros. Suppose that $\eta$ is a nonzero complex constant, $n, k(\geq 0)$ and $m(\geq 1)$ are integers such that $n \geq 2 k+m+6$. If $\left(f^{n}(z)\left(f^{m}(z)-1\right) f(z+\eta)\right)^{(k)}$ and $\left(g^{n}(z)\left(g^{m}(z)-1\right) g(z+\eta)\right)^{(k)}$ share “ $(\alpha(z), 2)$ ", then $f(z) \equiv \operatorname{tg}(z)$ where $t^{m}=1$. 
Theorem 2. Let $f(z)$ and $g(z)$ be two transcendental entire functions of finite order, and $\alpha(z)(\not \equiv$ $0, \infty)$ be a small function of both $f(z)$ and $g(z)$ with finitely many zeros. Suppose that $\eta$ is a nonzero complex constant, $n, k(\geq 0)$ and $m(\geq 1)$ are integers such that $n \geq 3 k+2 m+8$. If $\left(f^{n}(z)\left(f^{m}(z)-1\right) f(z+\eta)\right)^{(k)}$ and $\left(g^{n}(z)\left(g^{m}(z)-1\right) g(z+\eta)\right)^{(k)}$ share $(\alpha(z), 2)^{*}$, then $f(z) \equiv \operatorname{tg}(z)$ where $t^{m}=1$.

Theorem 3. Let $f(z)$ and $g(z)$ be two transcendental entire functions of finite order, and $\alpha(z)(\not \equiv$ $0, \infty)$ be a small function with respect to both $f(z)$ and $g(z)$ with finitely many zeros. Suppose that $\eta$ is a nonzero complex constant, $n, k(\geq 0)$ and $m(\geq 1)$ are integers such that $n \geq 5 k+$ $4 m+12$. If $\bar{E}_{2)}\left(\alpha(z),\left(f^{n}(z)\left(f^{m}(z)-1\right) f(z+\eta)\right)^{(k)}\right)=\bar{E}_{2)}\left(\alpha(z),\left(g^{n}(z)\left(g^{m}(z)-1\right) g(z+\eta)\right)^{(k)}\right)$, then $f(z) \equiv \operatorname{tg}(z)$ where $t^{m}=1$.

Remark 1. Since Theorems $\mathrm{H}-\mathrm{J}$ are the special cases of Theorems $1-3$ respectively for $k=0$, Theorems 1-3 improve and extend Theorems H-J respectively.

\section{Lemmas}

In this section, we state some lemmas which will be needed in the sequel. We denote by $H$ the following function:

$$
H=\left(\frac{F^{\prime \prime}}{F^{\prime}}-\frac{2 F^{\prime}}{F-1}\right)-\left(\frac{G^{\prime \prime}}{G^{\prime}}-\frac{2 G^{\prime}}{G-1}\right),
$$

where $F$ and $G$ are nonconstant meromorphic functions defined in the complex plane $\mathbb{C}$.

Lemma 1 ([4]). Let $f(z)$ be a meromorphic function of order $\rho(f)<\infty$, and let $\eta$ be a nonzero complex constant. Then for each $\varepsilon>0$, we have

$$
T(r, f(z+\eta))=T(r, f)+O\left\{r^{\rho(f)-1+\varepsilon}\right\}+O\{\log r\} .
$$

Lemma 2 ([3]). Let $f(z)$ be an entire function of order $\rho(f)<\infty$, and $F=f^{n}(z)\left(f^{m}(z)-1\right) f(z+$ $\eta)$. Then

$$
T(r, F)=(n+m+1) T(r, f)+O\left\{r^{\rho(f)-1+\varepsilon}\right\}+S(r, f) .
$$

Lemma 3 ([26]). Let $f$ be a nonconstant meromorphic function, and $p, k$ be positive integers. Then

$$
\begin{aligned}
& N_{p}\left(r, 0 ; f^{(k)}\right) \leq T\left(r, f^{(k)}\right)-T(r, f)+N_{p+k}(r, 0 ; f)+S(r, f), \\
& N_{p}\left(r, 0 ; f^{(k)}\right) \leq k \bar{N}(r, \infty ; f)+N_{p+k}(r, 0 ; f)+S(r, f) .
\end{aligned}
$$


Lemma 4 ([1]). Let $F$ and $G$ be two nonconstant meromorphic functions that share " $(1,2)$ " and $H \not \equiv 0$. Then

$T(r, F) \leq N_{2}(r, 0 ; F)+N_{2}(r, 0 ; G)+N_{2}(r, \infty ; F)+N_{2}(r, \infty ; G)-\sum_{p=3}^{\infty} \bar{N}\left(r, 0 ; \frac{G^{\prime}}{G} \mid \geq p\right)+S(r, F)+S(r, G)$, and the same inequality holds for $T(r, G)$.

Lemma 5 ([1]). Let $F$ and $G$ be two nonconstant meromorphic functions that share $(1,2)^{*}$ and $H \not \equiv 0$. Then

$T(r, F) \leq N_{2}(r, 0 ; F)+N_{2}(r, 0 ; G)+N_{2}(r, \infty ; F)+N_{2}(r, \infty ; G)+\bar{N}(r, 0 ; F)+\bar{N}(r, \infty ; F)-m(r, 1 ; G)+$ $S(r, F)+S(r, G)$,

and the same inequality is true for $T(r, G)$.

Lemma 6 ([16]). Let $F$ and $G$ be two nonconstant entire functions, and $p \geq 2$ be an integer. If $\bar{E}_{p)}(1, F)=\bar{E}_{p)}(1, G)$ and $H \not \equiv 0$, then

$T(r, F) \leq N_{2}(r, 0 ; F)+N_{2}(r, 0 ; G)+2 \bar{N}(r, 0 ; F)+\bar{N}(r, 0 ; G)+S(r, F)+S(r, G)$,

and the same inequality holds for $T(r, G)$.

Lemma 7. Let $f$ and $g$ be two entire functions, $n(\geq 1), m(\geq 1), k(\geq 0)$ be integers, and let

$$
F=\left(f^{n}(z)\left(f^{m}(z)-1\right) f(z+\eta)\right)^{(k)}, G=\left(g^{n}(z)\left(g^{m}(z)-1\right) g(z+\eta)\right)^{(k)} .
$$

If there exists nonzero constants $c_{1}$ and $c_{2}$ such that $\bar{N}\left(r, c_{1} ; F\right)=\bar{N}(r, 0 ; G)$ and $\bar{N}\left(r, c_{2} ; G\right)=$ $\bar{N}(r, 0 ; F)$, then $n \leq 2 k+m+3$.

Proof. We put $F_{1}=f^{n}(z)\left(f^{m}(z)-1\right) f(z+\eta)$ and $G_{1}=g^{n}(z)\left(g^{m}(z)-1\right) g(z+\eta)$. By the second fundamental theorem of Nevanlinna we have

$$
\begin{aligned}
T(r, F) & \leq \bar{N}(r, 0 ; F)+\bar{N}\left(r, c_{1} ; F\right)+S(r, F) \\
& \leq \bar{N}(r, 0 ; F)+\bar{N}(r, 0 ; G)+S(r, F) .
\end{aligned}
$$

Using (2.1), (2.2), (2.3) and Lemmas 1 and 2 we obtain

$$
\begin{aligned}
(n+m+1) T(r, f) \leq & T(r, F)-\bar{N}(r, 0 ; F)+N_{k+1}\left(r, 0 ; F_{1}\right)+S(r, f) \\
\leq & \bar{N}(r, 0 ; G)+N_{k+1}\left(r, 0 ; F_{1}\right)+S(r, f) \\
\leq & N_{k+1}\left(r, 0 ; F_{1}\right)+N_{k+1}\left(r, 0 ; G_{1}\right)+S(r, f)+S(r, g) \\
\leq & (k+1)(\bar{N}(r, 0 ; f)+\bar{N}(r, 0 ; g))+N\left(r, 1 ; f^{m}\right)+N\left(r, 1 ; g^{m}\right) \\
& +N(r, 0 ; f(z+\eta))+N(r, 0 ; g(z+\eta))+S(r, f)+S(r, g) \\
\leq & (k+m+2)(T(r, f)+T(r, g))+O\left\{r^{\rho(f)-1+\varepsilon}\right\} \\
& +O\left\{r^{\rho(g)-1+\varepsilon}\right\}+S(r, f)+S(r, g) .
\end{aligned}
$$


Similarly

$$
\begin{aligned}
(n+m+1) T(r, g) \leq & (k+m+2)(T(r, f)+T(r, g))+O\left\{r^{\rho(f)-1+\varepsilon}\right\} \\
& +O\left\{r^{\rho(g)-1+\varepsilon}\right\}+S(r, f)+S(r, g) .
\end{aligned}
$$

Combining (2.4) and (2.5) we obtain

$$
(n-2 k-m-3)(T(r, f)+T(r, g)) \leq O\left\{r^{\rho(f)-1+\varepsilon}\right\}+O\left\{r^{\rho(g)-1+\varepsilon}\right\}+S(r, f)+S(r, g),
$$

which gives $n \leq 2 k+m+3$. This proves the lemma.

Lemma 8 ([2]). Let $f(z)$ and $g(z)$ be two transcendental entire functions of finite order, and let $\eta$ be a nonzero complex constant, and $n, m$ be positive integers such that $n \geq m+5$. If

$$
f^{n}(z)\left(f^{m}(z)-1\right) f(z+\eta) \equiv g^{n}(z)\left(g^{m}(z)-1\right) g(z+\eta)
$$

then $f(z) \equiv \operatorname{tg}(z)$, where $t$ is a constant satisfying $t^{m}=1$

Note 1. Though the authors [2] claimed that the result holds for $n \geq m+6$, from the proof it is easily seen that the result holds if $n \geq m+5$.

\section{Proof of the Theorems}

Proof of Theorem 1. Let $F=\frac{F_{1}^{(k)}}{\alpha(z)}$ and $G=\frac{G_{1}^{(k)}}{\alpha(z)}$ where $F_{1}=f^{n}(z)\left(f^{m}(z)-1\right) f(z+\eta)$ and $G_{1}=$ $g^{n}(z)\left(g^{m}(z)-1\right) g(z+\eta)$. Then $F$ and $G$ are transcendental meromorphic functions that share “ $(1,2)$ ” except the zeros and poles of $\alpha(z)$. From Lemma 2 we see that

$$
\begin{aligned}
& T\left(r, F_{1}\right)=(n+m+1) T(r, f)+O\left\{r^{\rho(f)-1+\varepsilon}\right\}+S(r, f), \\
& T\left(r, G_{1}\right)=(n+m+1) T(r, g)+O\left\{r^{\rho(g)-1+\varepsilon}\right\}+S(r, g) .
\end{aligned}
$$

If possible, we may assume that $H \not \equiv 0$. Using (2.1), (3.1) and Lemma 2 we get

$$
\begin{aligned}
N_{2}(r, 0 ; F) & \leq N_{2}\left(r, 0 ;\left(F_{1}\right)^{(k)}\right)+S(r, f) \\
& \leq T\left(r,\left(F_{1}\right)^{(k)}\right)-(n+m+1) T(r, f)+N_{k+2}\left(r, 0 ; F_{1}\right)+S(r, f) \\
& \leq T(r, F)-(n+m+1) T(r, f)+N_{k+2}\left(r, 0 ; F_{1}\right)+S(r, f) .
\end{aligned}
$$

From this we get

$$
(n+m+1) T(r, f) \leq T(r, F)-N_{2}(r, 0 ; F)+N_{k+2}\left(r, 0 ; F_{1}\right)+S(r, f) .
$$

Also by (2.2) we obtain

$$
N_{2}(r, 0 ; F) \leq N_{2}\left(r, 0 ;\left(F_{1}\right)^{(k)}\right)+S(r, f)
$$




$$
\leq N_{k+2}\left(r, 0 ; F_{1}\right)+S(r, f) .
$$

Similarly,

$$
N_{2}(r, 0 ; G) \leq N_{k+2}\left(r, 0 ; G_{1}\right)+S(r, g) .
$$

Using (3.4), (3.5) and Lemmas 1 and 4 we obtain from (3.3)

$$
\begin{aligned}
(n+m+1) T(r, f) \leq & N_{2}(r, 0 ; G)+N_{2}(r, \infty ; F)+N_{2}(r, \infty ; G)+N_{k+2}\left(r, 0 ; F_{1}\right) \\
& +S(r, f)+S(r, g) \\
\leq & N_{k+2}\left(r, 0 ; F_{1}\right)+N_{k+2}\left(r, 0 ; G_{1}\right)+S(r, f)+S(r, g) \\
\leq & (k+m+3)\{T(r, f)+T(r, g)\}+O\left\{r^{\rho(f)-1+\varepsilon}\right\} \\
& +O\left\{r^{\rho(g)-1+\varepsilon}\right\}+S(r, f)+S(r, g) .
\end{aligned}
$$

In a similar manner we obtain

$$
\begin{aligned}
(n+m+1) T(r, g) \leq & (k+m+3)\{T(r, f)+T(r, g)\}+O\left\{r^{\rho(f)-1+\varepsilon}\right\} \\
& +O\left\{r^{\rho(g)-1+\varepsilon}\right\}+S(r, f)+S(r, g) .
\end{aligned}
$$

(3.6) and (3.7) together yields

$$
\begin{aligned}
(n-2 k-m-5)\{T(r, f)+T(r, g)\} \leq & O\left\{r^{\rho(f)-1+\varepsilon}\right\}+O\left\{r^{\rho(g)-1+\varepsilon}\right\} \\
& +S(r, f)+S(r, g),
\end{aligned}
$$

a contradiction with the assumption that $n \geq 2 k+m+6$. Therefore we must have $H=0$. Then

$$
\left(\frac{F^{\prime \prime}}{F^{\prime}}-\frac{2 F^{\prime}}{F-1}\right)-\left(\frac{G^{\prime \prime}}{G^{\prime}}-\frac{2 G^{\prime}}{G-1}\right)=0
$$

Integrating both side twice we get from above

$$
\frac{1}{F-1}=\frac{A}{G-1}+B
$$

where $A(\neq 0)$ and $B$ are constants. From (3.8) it is clear that $F, G$ share $1 \mathrm{CM}$ and hence they share " $(1,2)$ ". Therefore $n \geq 2 k+m+6$. We now discuss the following three cases separately.

Case 1. Suppose that $B \neq 0$ and $A=B$. Then from (3.8) we obtain

$$
\frac{1}{F-1}=\frac{B G}{G-1} \text {. }
$$

If $B=-1$, then from (3.9) we obtain $F G=1$. Then

$$
\left(f^{n}(z)\left(f^{m}(z)-1\right) f(z+\eta)\right)^{(k)}\left(g^{n}(z)\left(g^{m}(z)-1\right) g(z+\eta)\right)^{(k)}=\alpha^{2} .
$$


Since the number of zeros of $\alpha(z)$ is finite, it follows that $f$ as well as $g$ has finitely many zeros. We put $f(z)=h(z) e^{\beta(z)}$, where $h(z)$ is a nonzero polynomial and $\beta(z)$ is a nonconstant polynomial. Now replacing $\beta(z+\eta)$ by $\gamma(z)$ and $h(z+\eta)$ by $\mu(z)$ we deduce that

$$
\begin{aligned}
\left(f^{n}(z)\right. & \left.\left(f^{m}(z)-1\right) f(z+\eta)\right)^{(k)} \\
= & \left(h^{n}(z) e^{n \beta(z)}\left(h^{m}(z) e^{m \beta(z)}-1\right) h(z+\eta) e^{\beta(z+\eta)}\right)^{(k)} \\
= & \left(h^{n}(z) \mu(z) e^{n \beta(z)+\gamma(z)}\left(h^{m}(z) e^{m \beta(z)}-1\right)\right)^{(k)} \\
= & \left(h^{n+m}(z) \mu(z) e^{(n+m) \beta(z)+\gamma(z)}-h^{n}(z) \mu(z) e^{n \beta(z)+\gamma(z)}\right)^{(k)} \\
= & e^{(n+m) \beta(z)+\gamma(z)} P_{1}\left(\beta(z), \gamma(z), h(z), \mu(z), \ldots, \beta^{(k)}(z), \gamma^{(k)}(z), h^{(k)}(z), \mu^{(k)}(z)\right) \\
& -e^{n \beta(z)+\gamma(z)} P_{2}\left(\beta(z), \gamma(z), h(z), \mu(z), \ldots, \beta^{(k)}(z), \gamma^{(k)}(z), h^{(k)}(z), \mu^{(k)}(z)\right) \\
= & e^{n \beta(z)+\gamma(z)}\left(P_{1} e^{m \beta(z)}-P_{2}\right) .
\end{aligned}
$$

Obviously $P_{1} e^{m \beta(z)}-P_{2}$ has infinite number of zeros, which contradicts with the fact that $g$ is an entire function.

If $B \neq-1$, from (3.9), we have $\frac{1}{F}=\frac{B G}{(1+B) G-1}$ and so $\bar{N}\left(r, \frac{1}{1+B} ; G\right)=\bar{N}(r, 0 ; F)$. Using (2.1), (2.2), (3.2) and the second fundamental theorem of Nevanlinna, we deduce that

$$
\begin{aligned}
T(r, G) & \leq \bar{N}(r, 0 ; G)+\bar{N}\left(r, \frac{1}{1+B} ; G\right)+\bar{N}(r, \infty ; G)+S(r, G) \\
& \leq \bar{N}(r, 0 ; F)+\bar{N}(r, 0 ; G)+\bar{N}(r, \infty ; G)+S(r, G) \\
& \leq N_{k+1}\left(r, 0 ; F_{1}\right)+T(r, G)+N_{k+1}\left(r, 0 ; G_{1}\right)-(n+m+1) T(r, g)+S(r, g) .
\end{aligned}
$$

This gives

$$
\begin{aligned}
(n+m+1) T(r, g) \leq & (k+m+2)\{T(r, f)+T(r, g)\}+O\left\{r^{\rho(f)-1+\varepsilon}\right\} \\
& +O\left\{r^{\rho(g)-1+\varepsilon}\right\}+S(r, g) .
\end{aligned}
$$

Thus we obtain

$$
\begin{aligned}
(n-2 k-m-3)\{T(r, f)+T(r, g)\} \leq & O\left\{r^{\rho(f)-1+\varepsilon}\right\}+O\left\{r^{\rho(g)-1+\varepsilon}\right\} \\
& +S(r, f)+S(r, g),
\end{aligned}
$$

a contradiction since $n \geq 2 k+m+6$.

Case 2. Let $B \neq 0$ and $A \neq B$. Then from (3.8) we get $F=\frac{(B+1) G-(B-A+1)}{B G+(A-B)}$ and $\operatorname{so} \bar{N}\left(r, \frac{B-A+1}{B+1} ; G\right)=$ $\bar{N}(r, 0 ; F)$. Arguing similarly as in case 1 we arrive at a contradiction. 
Case 3. Let $B=0$ and $A \neq 0$. Then from (3.8) we get $F=\frac{G+A-1}{A}$ and $G=A F-(A-1)$. If $A \neq 1$, it follows that $\bar{N}\left(r, \frac{A-1}{A} ; F\right)=\bar{N}(r, 0 ; G)$ and $\bar{N}(r, 1-A ; G)=\bar{N}(r, 0 ; F)$. Now applying Lemma 7 it can be shown that $n \leq 2 k+m+3$, a contradiction. Thus $A=1$ and then $F=G$. Then

$$
\left(f^{n}(z)\left(f^{m}(z)-1\right) f(z+\eta)\right)^{(k)}=\left(g^{n}(z)\left(g^{m}(z)-1\right) g(z+\eta)\right)^{(k)} .
$$

Integrating once we obtain

$$
\left(f^{n}(z)\left(f^{m}(z)-1\right) f(z+\eta)\right)^{(k-1)}=\left(g^{n}(z)\left(g^{m}(z)-1\right) g(z+\eta)\right)^{(k-1)}+c_{k-1},
$$

where $c_{k-1}$ is a constant. If $c_{k-1} \neq 0$, using Lemma 7 it follows that $n \leq 2 k+m+1$, a contradiction. Hence $c_{k-1}=0$. Repeating the process $k$-times, we deduce that

$$
f^{n}(z)\left(f^{m}(z)-1\right) f(z+\eta)=g^{n}(z)\left(g^{m}(z)-1\right) g(z+\eta)
$$

which by Lemma 8 gives $f=t g$, where $t$ is a constant satisfying $t^{m}=1$

This completes the proof of Theorem 1 .

Proof of Theorem 2. Let $F, G, F_{1}$ and $G_{1}$ be defined as in Theorem 1 . Then $F$ and $G$ are transcendental meromorphic functions that share $(1,2)^{*}$ except the zeros and poles of $\alpha(z)$. Let $H \not \equiv 0$. Then using (2.2) for $p=1$, (3.5) and Lemmas 1 and 5 we obtain from (3.3)

$$
\begin{aligned}
(n+m+1) T(r, f) \leq & N_{2}(r, 0 ; G)+N_{2}(r, \infty ; F)+N_{2}(r, \infty ; G)+\bar{N}(r, 0 ; F) \\
& +\bar{N}(r, \infty ; F)+N_{k+2}\left(r, 0 ; F_{1}\right)+S(r, f)+S(r, g) \\
\leq & N_{k+2}\left(r, 0 ; F_{1}\right)+N_{k+2}\left(r, 0 ; G_{1}\right)+N_{k+1}\left(r, 0 ; F_{1}\right)+S(r, f)+S(r, g) \\
\leq & (2 k+2 m+5) T(r, f)+(k+m+3) T(r, g)+O\left\{r^{\rho(f)-1+\varepsilon}\right\} \\
& +O\left\{r^{\rho(g)-1+\varepsilon}\right\}+S(r, f)+S(r, g) .
\end{aligned}
$$

In a similar manner we obtain

$$
\begin{aligned}
(n+m+1) T(r, g) \leq & (2 k+2 m+5) T(r, g)+(k+m+3) T(r, f)+O\left\{r^{\rho(f)-1+\varepsilon}\right\} \\
& +O\left\{r^{\rho(g)-1+\varepsilon}\right\}+S(r, f)+S(r, g) .
\end{aligned}
$$

From (3.10) and (3.11) we get

$$
\begin{aligned}
(n-3 k-2 m-7)\{T(r, f)+T(r, g)\} \leq & O\left\{r^{\rho(f)-1+\varepsilon}\right\}+O\left\{r^{\rho(g)-1+\varepsilon}\right\} \\
& +S(r, f)+S(r, g),
\end{aligned}
$$

contradicting with the fact that $n \geq 3 k+2 m+8$. Thus we must have $H=0$. Then the result follows from the proof of Theorem 1 . This completes the proof of Theorem 2 . 
Proof of Theorem 3. Let $F, G, F_{1}$ and $G_{1}$ be similar as in Theorem 1 . Then $F$ and $G$ are transcendental meromorphic functions such that $\bar{E}_{2)}(1, F)=\bar{E}_{2)}(1, G)$ except the zeros and poles of $\alpha(z)$. Let $H \not \equiv 0$. Then by (2.2), (3.5) and Lemmas 1 and 6 we obtain from (3.3)

$$
\begin{aligned}
(n+m+1) T(r, f) \leq & N_{2}(r, 0 ; G)+2 \bar{N}(r, 0 ; F)+\bar{N}(r, 0 ; G)+N_{k+2}\left(r, 0 ; F_{1}\right) \\
& +S(r, f)+S(r, g) \\
\leq & N_{k+2}\left(r, 0 ; F_{1}\right)+N_{k+2}\left(r, 0 ; G_{1}\right)+2 N_{k+1}\left(r, 0 ; F_{1}\right)+N_{k+1}\left(r, 0 ; G_{1}\right) \\
& +S(r, f)+S(r, g) \\
\leq & (3 k+3 m+7) T(r, f)+(2 k+2 m+5) T(r, g)+O\left\{r^{\rho(f)-1+\varepsilon}\right\} \\
& +O\left\{r^{\rho(g)-1+\varepsilon}\right\}+S(r, f)+S(r, g) .
\end{aligned}
$$

Similarly,

$$
\begin{aligned}
(n+m+1) T(r, g) \leq & (3 k+3 m+7) T(r, g)+(2 k+2 m+5) T(r, f)+O\left\{r^{\rho(f)-1+\varepsilon}\right\} \\
& +O\left\{r^{\rho(g)-1+\varepsilon}\right\}+S(r, f)+S(r, g) .
\end{aligned}
$$

Combining (3.12) and (3.13) we obtain

$$
(n-5 k-4 m-11)\{T(r, f)+T(r, g)\} \leq O\left\{r^{\rho(f)-1+\varepsilon}\right\}+O\left\{r^{\rho(g)-1+\varepsilon}\right\}+S(r, f)+S(r, g),
$$

a contradiction with the assumption that $n \geq 5 k+4 m+12$. Thus $H=0$ and the rest of the theorem follows from the proof of Theorem 1 . This completes the proof of Theorem 3 .

Open Problems. In the paper, we give two open questions for further research.

Question 4. What can we get if we consider transcendental meromorphic functions in Theorems $1-3$ ?

Question 5. Can we relax the lower bound of $n$ in Theorems 1-3?

\section{Acknowledgement}

The authors are grateful to the referee for his/her valuable suggestions/comments towards the improvement of the paper.

\section{References}

[1] A. Banerjee and S. Mukherjee, Uniqueness of meromorphic functions concerning differential monomials sharing the same value, Bull. Math. Soc. Sci. Math. Roum. Nouv. Ser., 50 (2007), 191-206.

[2] S. S. Bhoosnurmath and S. R. Kabbur, Value distribution and uniqueness theorems for difference of entire and meromorphic functions, Int. J. Anal. Appl., 2 (2013), 124-136. 
[3] M. R. Chen and Z. X. Chen, Properties of difference polynomials of entire functions with finite order, Chinese Annals of Mathematics, 33 (2012), 359-374.

[4] Y. M. Chiang and S. J. Feng, On the Nevanlinna characteristic of $f(z+\eta)$ and difference equations in the complex plane, Ramanujan J., 16 (2008), 105-129.

[5] M. L. Fang and W. Hong, A unicity theorem for entire functions concerning differential polynomials, Indian J. Pure Appl. Math., 32 (2001), 1343-1348.

[6] M. L. Fang and X. H. Hua, Entire functions that share one value, J. Nanjing Univ. Math. Biquartely, 13 (1996), 44-48.

[7] R. G. Halburd and R. J. Korhonen, Nevanlinna theory for the difference operator, Ann. Acad. Sci. Fenn. Math., 31 (2006), 463-478.

[8] R. G. Halburd and R. J. Korhonen, Difference analogue of the lemma on the logarithmic derivative with application to difference equations, J. Math. Anal. Appl., 314 (2006), 477-487.

[9] W. K. Hayman, Meromorphic Functions, The Clarendon Press, Oxford, 1964.

[10] I. Lahiri, Value distribution of certain differential polynomials, Int. J. Math. Math. Sci., 28 (2001), 83-91.

[11] I. Lahiri, Weighted value sharing and uniqueness of meromorphic functions, Complex Var. Theory Appl., 46 (2001), 241-253.

[12] I. Laine, Nevanlinna Theory and Complex Differential Equations, Walter de Gruyter, Berlin/Newyork, 1993.

[13] I. Laine and C. C Yang, Value distribution of difference polynomials, Proc. Japan Acad. Ser. A Math. Sci., 83 (2007), 148-151.

[14] S. H. Lin and W. C. Lin, Uniqueness of meromorphic functions concerning weakly weighted sharing, Kodai Math. J., 29 (2006), 269-280.

[15] W. C. Lin and H. X. Yi, Uniqueness theorems for meromorphic functions concerning fixed points, Complex Var. Theory Appl., 49 (2004), 793-806.

[16] X. Q. Lin and W. C. Lin, Uniqueness of entire functions sharing one value, Acta Math. Sci., Ser. B. Engl. Ed., 31 (2011), 1062-1076.

[17] K. Liu and L. Z. Yang, Value distribution of the difference operator, Arch. Math. Basel, 92 (2009), $270-278$.

[18] C. Meng, Uniqueness of entire functions concerning difference polynomials, Math. Bohemica, 139 (2014), 8997.

[19] X. G. Qi, L. Z. Yang and K. Liu, Uniqueness and periodicity of meromorphic functions concerning the difference operator, Computers and Mathematics with Applications, 60 (2010), 1739-1746.

[20] P. Sahoo, Unicity theorem for entire functions sharing one value, Filomat, 27 (2013), 797-809.

[21] P. Sahoo, Uniqueness of entire functions related to difference polynomials, Commu. Math. Stat., 3 (2015), 227238.

[22] P. Sahoo and S. Seikh, Meromorphic functions whose certain differential polynomials share a small function with finite weight, Analysis (Munich), 33 (2013), 143-157.

[23] C. C Yang and X. H. Hua, Uniqueness and value sharing of meromorphic functions, Ann. Acad. Sci. Fenn. Math., 22 (1997), 395-406.

[24] C. C. Yang and H. X. Yi, Uniqueness Theory of Meromorphic Functions, Kluwer, Dordrecht, 2003.

[25] J. L. Zhang, Value distribution and shared sets of difference of meromorphic functions, J. Math. Anal. Appl., 367 (2010), 401-408.

[26] J. L. Zhang and L. Z. Yang, Some results related to a conjecture of R. Bruck, J. Inequal. Pure Appl. Math., 8 (2007), Art. 18.

Department of Mathematics, University of Kalyani, West Bengal-741235, India.

E-mail: sahoopulak@yahoo.com,sahoopulak1@gmail.com

Department of Mathematics, HooghlyWomen's College, Hooghly, West Bengal-712103, India.

E-mail: gdb.math@gmail.com 